Cahiers de la recherche sur les droits fondamentaux

$6 \mid 2008$

Pouvoirs exceptionnels et droits fondamentaux

\title{
La loi du 5 mars 2007, le droit pénal et l'Internet
}

Sylvain Jacopin

\section{OpenEdition}

Journals

Édition électronique

URL : https://journals.openedition.org/crdf/6902

DOI : $10.4000 /$ crdf.6902

ISSN : 2264-1246

Éditeur

Presses universitaires de Caen

Édition imprimée

Date de publication : 31 décembre 2008

Pagination : 149-160

ISBN : 978-2-84133-259-5

ISSN : 1634-8842

Référence électronique

Sylvain Jacopin, «La loi du 5 mars 2007, le droit pénal et l'Internet », Cahiers de la recherche sur les droits fondamentaux [En ligne], 6 | 2008, mis en ligne le 11 décembre 2020, consulté le 14 novembre 2022. URL : http://journals.openedition.org/crdf/6902 ; DOI : https://doi.org/10.4000/crdf.6902 


\title{
La loi du 5 mars 2007, le droit pénal et l'Internet
}

\author{
Sylvain JACOPIN \\ Maître de conférences à l'Université de Caen Basse-Normandie \\ Membre du Centre de recherches en droit privé
}

I. Un compromis entre deux logiques opposées: le happy slapping

A. Une nouvelle infraction de prévention : la logique de répression

1. L'enregistrement

2. La diffusion

3. Les violences concernées

B. L'exception à la répression : un fait justificatif né d'une logique de protection

1. L'exception d'information

2. L'exception probatoire

II. Les impasses du compromis: le dispositif de protection des mineurs au service d'une meilleure répression des acteurs de la prévention

A. De nouvelles obligations à la charge des intermédiaires techniques

1. La lutte contre l'incitation à la violence et les atteintes à la dignité

2. La lutte contre les activités illicites de jeu d'argent

3. L'autorité chargée de prononcer les interdictions et de fixer les caractéristiques de la signalétique

B. De nouvelles prérogatives pour des acteurs étrangers aux réseaux

1. Constats et preuves des infractions par les cyber-patrouilleurs

2. Gel des fonds des organisateurs de jeux illicites par les établissements financiers

III. Conclusion

En quelques années, le législateur s'est successivement intéressé aux droits des victimes (loi du 4 mars 2002 complétant la loi du 15 juin 2000, JO des 4 et 5 mars 2002, p. 4169-4171), aux grandes orientations de la justice (loi du 9 septembre 2002, JO du 10 septembre 2002, p. 14934), à la sécurité intérieure (loi du 18 mars 2003, JO du 19 mars 2003, p. 4761), à l'adaptation de la justice aux évolutions de la criminalité (loi du 9 mars 2004, JO du 10 mars 2004, p. 4567), au traitement de la récidive (loi du 12 décembre 2005, JO du 13 décembre 2005, et loi du 10 août 2007, JO du 11 août 2007), ou encore au terrorisme (loi du 23 janvier 2006, JO du 24 janvier 2006, p. 1129).
La prévention est le nouvel objectif poursuivi par le législateur dans cette loi du 5 mars 2007. Voilà une date symbolique, puisque le même jour ont été votées la loi relative à l'équilibre de la procédure pénale ( $J O$ du 6 mars 2007, p. 4206), celle relative à la responsabilité des magistrats (JO du 6 mars 2007, p. 4184), celle sur la protection juridique des majeurs protégés (JO du 7 mars 2007, p. 4325) et celle qui fait aujourd'hui l'objet de notre étude (loi $\mathrm{n}^{0}$ 2007-297 du 5 mars 2007 relative à la prévention de la délinquance, JO du 7 mars 2007, p. 4347).

Comment se manifeste la prévention en droit pénal ? La prévention juridique résulte d'incriminations de 
prévention qui tendent à arrêter une activité dangereuse alors qu'il en est encore temps. Ainsi, dans cette famille d'infraction, on peut distinguer les infractions qui saisissent une activité criminelle principale aux premiers stades de son développement et celles qui visent une activité criminelle simplement accessoire en écartant exceptionnellement les règles de la complicité. Entrent dans la première catégorie les délits qui tendent à stopper une activité criminelle avant qu'elle n'ait causé un dommage. Ces délits se présentent sous des formes diverses selon qu'ils se situent, dans l'iter criminis, au niveau de la résolution criminelle (le délit d'intention telle l'association de malfaiteurs, art. 450 $\mathrm{CP})$, à celui des actes préparatoires (le délit obstacle, dont l'exemple type, sous l'empire de l'ancien CP, est la fabrication de fausse clef, art. $399 \mathrm{CP}$, et qui se définit comme l'incrimination d'un acte préparatoire à une infraction de base donnée ${ }^{1}$ ) ou à celui du commencement d'exécution (délits formels, lesquels se situent au niveau du commencement d'exécution ${ }^{2}$ ). La seconde catégorie concerne des infractions qui saisissent un acte accessoire mais unies par un lien de connexité à une infraction principale et ne pouvant être punissable par le biais de la théorie du concert frauduleux, tantôt au titre de la coaction, tantôt au titre de la complicité. Le législateur incrimine alors parfois directement certains actes, soit d'instigation ou de provocation $^{3}$, soit consécutifs à une infraction punissable (recel $)^{4}$. Une situation extrême peut également être trouvée: des délits accessoires peuvent être incriminés alors que le délit de base n'existe pas dans les textes. On voit alors se développer une famille d'infractions de prévention sans délit de base. Il en est ainsi dans le domaine de la prostitution ${ }^{5}$, ce qui suppose au minimum que ladite activité, sans être punissable en soi pénalement, soit illicite.

La prévention matérielle consiste à dresser des obstacles physiques (bandes rugueuses à l'approche d'un carrefour) ou psychologiques (inscription «le tabac tue» sur les paquets de cigarettes) pour contraindre ou inciter les gens à se conduire de manière conforme à l'intérêt général et à leur intérêt propre.

Comment se manifeste la prévention dans la loi du 5 mars 2007 ?
Dans les travaux préparatoires de la loi de prévention, il est précisé que la politique de prévention a pour sens la lutte contre l'insécurité. Prioritairement dirigée vers les mineurs, elle se fonde sur un pilier central : l'éducation. Paradoxalement, les moyens mis en œuvre s'articulent autour de la systématisation de la réponse pénale visant à réduire le passage à l'acte. C'est ainsi un pan entier de la logique juridique applicable au droit pénal des mineurs qui est ici inversé. Alors que le droit pénal des mineurs est traditionnellement considéré comme le droit de l'expérimentation pour son éventuelle application au droit des majeurs ${ }^{6}$, il tend aujourd'hui à se rapprocher du droit des majeurs. En effet, son régime devient progressivement de droit commun alors même que les mineurs continuent à être soumis à l'ordonnance de 1945, un texte marqué par son caractère éminemment éducatif. Le Conseil constitutionnel s'efforce d'ailleurs bon gré mal gré de freiner ce mouvement en décidant que certains principes propres au droit des mineurs sont des principes fondamentaux reconnus par les lois de la république (Décision $n^{\circ} 2002-$ 461 DC, 29 août 2002). C'est le cas pour la priorité de l'action éducative ou celui de l'atténuation de la responsabilité des mineurs. Dans ce contexte (hasard du calendrier ?), il faut préciser que la loi de prévention sur la délinquance a été votée en même temps que celle sur la protection del'enfance. La coïncidence a sa valeur : les deux problématiques que sont l'enfance en danger et l'enfance délinquante sont historiquement liées. En 1945, a été votée l'ordonnance sur l'enfance délinquante là où aucun texte sur l'enfance en danger n'existait. En 1958, il a été donné mission aux juges des enfants de s'occuper de l'enfance en danger avec le souci d'éviter la délinquance chez les jeunes. La justice des mineurs s'en est trouvée transformée: au nom de la prévention, la justice pénale s'est axée sur la protection de l'enfance. La prévention de la délinquance semble enterrer aujourd'hui toute idée de la protection. La prévention se traduit par une réponse musclée à la délinquance juvénile. Que la répression poursuive un tel objectif n'est pas nouveau. Et l'article 132-24 CP, modifié par la loi du 12 décembre 2005, précise cette fonction de la peine. Mais, ce qui paraît intéressant de relever avec la loi, c'est qu'elle

1. L'intérêt de cette catégorie est double. D'une part, puisqu'elle est centrée sur la notion d'acte, contrairement aux délits d'intention, elle se situe au premier stade utile de l'incrimination des activités dangereuses pour la société. D'autre part, elle recouvre des actes n'entrant pas dans le domaine d'application de la théorie de la tentative, et ne pouvant dès lors relever que de la loi.

2. S'ils paraissent de la sorte entrer en concurrence avec la théorie de la tentative, ils s'en distinguent cependant nettement. Les délits formels, prévus cas par cas par le législateur, s'étagent en effet, selon la nature de chaque incrimination, du pur commencement d'exécution jusqu'à l'instant de raison qui précède l'exécution parfaite. La théorie de la tentative, en revanche, d'application jurisprudentielle, couvre en toute hypothèse l'ensemble de ce stade de l'iter criminis. Le législateur recourt principalement à une incrimination de ce type dans les hypothèses où l'acte à proscrire consiste dans le déclenchement d'un mécanisme qui, une fois mis en mouvement, échappe au contrôle de l'agent. Voir délit de risque causé à autrui, art. 223-1 CP.

3. Il s'agit d'appréhender l'instigation non suivie d'effets. L'article 221-5-1 CP incrimine, depuis 2004, le fait de faire à une personne des offres ou des promesses ou de lui proposer des dons, présents ou avantages quelconques afin qu'elle commette un assassinat ou un empoisonnement dans le cas où ce crime n'a été ni commis ni tenté.

4. Un individu peut ne s'associer à une infraction pénale qu'après sa commission, pour en tirer profit ou assister ses auteurs. Il s'agit là d'un acte de complicité a posteriori. Si cet acte est directement incriminé par le législateur, il devient un délit de conséquence. Il repose sur une condition préalable puisque son application ne peut être envisagée qu'à partir de faits antérieurs constitutifs d'un délit pénal.

5. La prostitution est une activité qui a été laissée hors champ du droit pénal. Pour autant, il existe un certain nombre de délits obstacles afférents à cette activité : le racolage (art. 225-10-1 CP), la fourniture de renseignements (art. 225-6 CP), d'un local (art. 225-10 CP), d'un véhicule (art. 225-10 CP), la gérance d'un établissement de prostitution (art. 225-10 CP), ou encore son financement (art. 225-10 CP).

6. Voir, par exemple, le principe de personnalisation, aujourd'hui inscrit à l'article 132-24 CP et contenu depuis déjà longtemps dans l'article 2 de l'ordonnance du 2 février 1945. 
revisite le concept de prévention en marquant le passage d'un modèle de traitement social à celui du contrôle social. Il suffit de constater l'aggravation de la répression et la nouvelle place donnée au maire de procéder à un rappel à la loi dans certaines circonstances (cf. dans ce volume la contribution de J.-M. Larralde). La proposition phare initiale était d'ailleurs d'abaisser la majorité pénale à 16 ans. La difficulté majeure était de concilier et de veiller à la cohérence de deux textes émanant de la même majorité. Or, le parcours parlementaire de chacune des deux lois atteste une logique différente dans un contexte politique marqué, opposition du point de vue technique, que ce soit sur le champ du secret professionnel, ou à propos de la cellule de signalement des enfants maltraités. Si la loi de protection de l'enfance a fait aussi l'objet d'un consensus, la loi sur la prévention de la délinquance a une genèse de quatre ans. Indéniablement, des pratiques nouvelles vont se développer. Fondamentalement, la logique de répression contredit la logique de protection. Les incohérences et les paradoxes naissent $a$ priori de cette confrontation de logiques que sous-entend une loi de prévention. Mais l'examen attentif des dispositions démontre une réelle volonté d'accentuer la répression.

L'exemple le plus significatif de la loi est certainement les dispositions en matière de cybercriminalité. La loi sur la prévention axe principalement ces dispositions sur la répression des violences par le biais d'Internet. Internet, objet d'une infraction pénale $e^{7}$, peut aussi être le moyen de commettre une infraction pénale ${ }^{8}$. Instrument de réalisation de l'infraction, Internet devient le vecteur d'atteintes au droit des personnes. C'est le principal objet de cette loi : s'être intéressé à cet aspect d'Internet. L'essentiel de ses dispositions invite ainsi tant les usagers que les acteurs concernés en lien avec les mineurs à la vigilance. Certaines infractions peuvent donc être facilitées par l'usage d'Internet, et un cas extrême mérite une attention particulière: le happy slapping. La loi du 5 mars 2007 ajoute une nouvelle catégorie au sein des infractions de prévention liée non pas à une activité criminelle en cours mais au caractère accessoire de l'activité appréhendée. On connaît déjà ces infractions de prévention (v. supra). Mais désormais - et la situation est nouvelle - le caractère accessoire d'une activité est appréhendé du simple fait de son caractère immoral dans son rapport à la commission de l'infraction de base (I). En même temps, dans un objectif de protection des mineurs, la loi a pour ambition de mobiliser les acteurs de l'Internet tout en leur faisant courir un risque pénal non négligeable (II). Et, dans les deux cas, les contradictions et les lacunes sont constamment présentes.

\section{Un compromis entre deux logiques opposées : le happy slapping}

L'article 44-VI de la loi crée dans une nouvelle section du CP intitulée «De l'enregistrement et de la diffu- sion d'images de violence » un article 223-33-3 composé de trois alinéas qui successivement sanctionnent l'enregistrement et la diffusion d'images de violences mais prévoient deux exceptions aux incriminations. Sans être spécifiques aux mineurs, les dispositions s'adressent principalement à eux.

Avec cette nouvelle disposition, il s'agit d'appréhender la pratique dite du happy slapping (joyeuse baffe). La situation consiste initialement à frapper plus ou moins violemment une personne et à filmer la scène (le plus souvent au moyen d'un téléphone mobile). Tout l'intérêt réside dans le fait de visionner la scène à volonté, mais aussi de la diffuser largement, notamment grâce à l'Internet (via des sites de partage de vidéos). Méconnue il y a quelques années, la pratique est essentiellement le fait de mineurs et de jeunes majeurs. Les premières affaires en France ont été largement médiatisées : le viol d'une collégienne à Nice fin 2005, dont les images avaient été montrées au sein de l'établissement scolaire par son auteur, ou l'agression, en avril 2006 à Porcheville, d'une enseignante par un élève tandis qu'un autre filmait la scène avec son téléphone mobile.

\section{A. Une nouvelle infraction de prévention: la logique de répression}

Les deux premiers alinéas instituent une distinction fondamentale entre le fait d'enregistrer et le fait de diffuser les images de violence. Une telle distinction est pertinente, les diffuseurs successifs d'une vidéo étant le plus souvent distincts de l'individu à l'origine de l'enregistrement. Instinctivement, il paraît plus répréhensible de filmer une scène de violence sans intervenir, le cas échéant, avec une abstention coupable que de diffuser une vidéo sans entretenir quelque rapport que ce soit ni avec les agresseurs ni avec la victime. D'où le choix du législateur quant à la structure juridique de chacune de ces deux infractions, laquelle permet, pour l'enregistrement, que la peine encourue suive celle prévue pour la violence concernée, alors que, pour la diffusion, la peine prévue est unique dans la mesure où, quelle que soit la violence, la sanction pénale est fixée à 5 ans et 75000 euros.

\section{L'enregistrement}

Initialement proposée comme circonstance aggravante d'un délit distinct, ce qui aurait posé des problèmes dans son application car elle n'aurait concerné que celui commettant les violences mais en aucun cas celui se bornant à les filmer, sauf à démontrer que ce dernier est complice de l'auteur, c'est-à-dire qu'il est l'instigateur de l'infraction, le comportement est puni comme acte particulier de complicité.

Le texte vise l'enregistrement par quelque moyen que ce soit, et quel qu'en soit le support. Il doit être réalisé sciemment, ce qui implique que celui qui enregistre par inadvertance ne pourra être considéré comme complice.

7. Loi du 6 janvier 1978, mod. par la loi du 6 août 2004, voir Étude A. Lepage, comm. Comm. Elctr. 2005, p. 33.

8. Pour une synthèse des enjeux d'Internet par rapport au droit pénal, voir A. Lepage, Droit pénal et Internet, la part de la tradition, l'œeuvre de l'innovation, AJ Pénal 2005, nº 6, p. 21. 
Et les images doivent être relatives à la commission des violences visées. Autrement dit, l'enregistrement d'une simple tentative est punissable. Par contre, ni la préparation de l'infraction ni la période postérieure à la consommation de l'infraction n'entrent dans le domaine de l'article 222-33-3 CP. Par exemple, filmer la victime d'actes de violence une fois leur auteur parti.

Le fait de considérer l'enregistrement comme un cas de complicité procède de la volonté de faire appliquer également les circonstances aggravantes encourues par les violences elles-mêmes. En effet, le système d'emprunt de criminalité imposé par l'article 121-6 CP prévoit que le complice est puni comme l'auteur induisant une communication des circonstances aggravantes réelles au complice, c'est-à-dire toutes les circonstances liées à l'accomplissement de l'acte, notamment celles relatives à la qualité de la victime.

Cette particularité brouille cependant les repères concernant la forme traditionnelle de la complicité. Définir ainsi un cas spécial de complicité semble bien peu orthodoxe dans le système juridique français ${ }^{9}$ qui fonctionne par une définition générale de la complicité à l'article 1217 du Code pénal. L'article 121-7 précise trois formes de participation matérielle du complice qui sont l'aide ou l'assistance, la provocation et les instructions. Est ajouté ici un nouvel acte de complicité : le fait d'enregistrer. Cette nouvelle disposition bouleverse la notion même de complicité car elle confond la notion populaire de complicité et la notion juridique : être complice au sens du droit pénal, c'est vouloir s'associer à une infraction, c'est avoir la volonté de concourir à celle-ci. C'est pourquoi la complicité requiert l'entente, la collusion, entre l'auteur et le complice. Ce dernier doit contribuer à la réalisation de l'infraction. L'acte de complicité à ce titre doit présenter un caractère de causalité avec l'infraction principale, car il rend possible la réalisation de celle-ci. Or, ce texte permet de considérer comme complice celui qui n'a aucun lien avec l'agresseur, qui se trouve être le simple témoin de l'agression et qui la filme. Ici, l'activité matérielle du complice n'a aucun rôle causal dans la commission de l'infraction. Ainsi, il y a une extension de la notion de complicité : la disposition marque le passage d'une complicité par volonté d'adhérer à une complicité par simple approbation, adhésion morale à l'illicite: celui qui filme est neutre dans la commission de l'infraction, mais non face à l'infraction. Cette conception correspond d'ailleurs à la volonté du législateur telle qu'elle est exprimée dans les travaux parlementaires. Un petit curieux qui, sans être lié à l'auteur de l'agression, enregistre l'infraction pour satisfaire sa propre perversité est assimilé à la complicité. Si ce comportement contraire à la moralité publique doit être sanctionné pénalement, sans doute aurait-il été plus judicieux de l'envisager de manière autonome par le biais de l'incrimination relative à l'exploitation des images de violence, en s'inspirant par exemple de la structure de l'article 227-33 CP relatif à l'exploitation de l'image d'un mineur. Ainsi, il convient de s'interroger sur la nécessité du texte pénal. Autrement dit, la question se pose de savoir s'il était possible d'appréhender le comportement d'enregistrement des violences par le biais de la complicité dans sa forme traditionnelle.

Hors les hypothèses où l'auteur de l'enregistrement est l'instigateur des violences, il aurait été tout à fait possible de retenir la complicité par aide ou assistance au sens de l'article 121-6 CP. Certes, une simple inaction de l'individu, alors que ce dernier aurait pu s'opposer à la réalisation de l'infraction, ne peut fonder les poursuites. La jurisprudence le rappelle régulièrement (solution classique). Pour autant, le juge pose des limites à ce principe en considérant que l'individu spectateur simplement passif peut avoir une adhésion morale à l'infraction et constituer une aide à l'égard de l'auteur : soit en vertu d'un accord passé avec l'auteur avant la commission de l'infraction (Cass. crim., 11 avril 1983), soit l'individu avait le pouvoir et le devoir d'agir en raison de sa profession (Cass. crim., 15 mai 1997), soit encore l'abstention correspond à une caution morale apportée à l'auteur. Dès lors, la seule présence sur les lieux qui se manifeste par une adhésion ou une assistance morale à l'auteur (comme un encouragement à commettre l'infraction) traduit une complicité punissable, et pas seulement une passivité. L'aide ainsi psychologique et morale se traduit comme une participation matérielle au délit. Il a été ainsi jugé que la seule présence d'un individu au sein d'un groupe d'agresseurs, par son adhésion pleinement à l'intention délictueuse du groupe, fortifie moralement les auteurs - d'où complicité punissable (Cass. crim., 11 janvier 1992). La même solution peut donc être appliquée pour l'individu accompagné d'une caméra..., l'enregistrement permettant alors de caractériser cette adhésion à l'intention délictueuse du groupe, tout en étant constitutif d'un encouragement pour les auteurs. C'est d'ailleurs le raisonnement adopté par la Cour d'appel de Versailles en date du 24 octobre 2006, qui a condamné pour complicité du délit de violences volontaires commises à l'occasion des entrées et sorties des élèves aux abords d'un établissement scolaire et éducatif, prévu par l'article 222-13 $11^{\circ} \mathrm{CP}$, l'élève mineur qui a eu directement connaissance du projet de l'auteur du délit de frapper un condisciple après la classe (ces violences ayant entraîné une incapacité totale de travail personnel n'excédant pas huit jours), et qui en outre, en filmant la scène à l'aide de son téléphone portable et donnant ainsi plus d'écho à la manifestation par l'auteur de sa supériorité physique, l'a invité à faire étalage de sa force: selon les juges, il a facilité l'infraction, et il y a bien aide morale volontaire.

Si les individus sont totalement extérieurs aux agresseurs, la solution n'est plus envisageable. Pour autant, là encore, il existe des dispositions applicables. Le Code pénal contient des incriminations assurant la protection de l'image de la personne. L'article 226-1 CP incrimine « le fait au moyen d'un procédé quelconque volontairement de porter atteinte à l'intimité de la vie privée d'autrui. $2^{\circ}$ en fixant, enregistrant, ou transmettant sans le consentement 
de celle-ci l'image d'une personne se trouvant dans un lieu privé ». Or, les faits de happy slapping se réalisant le plus souvent dans des lieux publics, la disposition s'applique rarement. Outre ces atteintes à la vie privée, des poursuites sur le fondement de non-assistance à personne en péril et de non-obstacle à la commission d'une infraction (223-6 CP) à l'encontre de celui qui enregistre la scène de violence sont concevables ${ }^{10}$. Encore faut-il qu'il n'y ait aucun risque sérieux pour soi-même ou pour les tiers.

\section{La diffusion}

La situation est cette fois-ci appréhendée comme une infraction autonome. Il s'agit d'un nouveau délit de conséquence. D'ailleurs, il a été considéré comme une forme de « recel » par le législateur. C’est bel et bien la diffusion par quelque moyen que ce soit qui est réprimée : par téléphones portables, par courrier électronique, par webblog... Encore faut-il la connaissance du contenu des images et la volonté de les diffuser. Voici une infraction concernant directement le droit de la communication qui emploie des termes, sinon étrangers à la matière, du moins très vagues. En effet, diffuser est un verbe entretenant un paradoxe malheureux en ce sens qu'il s'avère très parlant pour le commun des mortels et pourtant très incertain pour le juriste. Il aurait peut-être été plus exact d'employer des termes comme «mise à disposition du public » ou encore «communication au public» qui visent spécifiquement l'éditeur du message. Toutefois, il faut noter que le législateur l'a utilisé depuis déjà quelques années pour d'autres infractions (notamment le délit de diffusion d'images pédophiles de l'article 227-23 CP).

La création de ce délit de diffusion posera la question du recel. En effet, dans le cadre du délit de diffusion d'images pédophiles prévu à l'article $227-23 \mathrm{CP}$, la chambre criminelle de la Cour de cassation a eu l'occasion d'affirmer, sur le fondement de l'article 321-1 CP, que « le recel du délit de fixation, enregistrement ou transmission, en vue de leur diffusion, d'images pornographiques de mineurs est constitué lorsque lesdites images ont été conservées dans un fichier enregistré sur le disque dur d'un ordinateur » (Cass. crim., 28 septembre 2005, $\mathrm{n}^{\circ}$ 04-85 024). Dès lors, l'internaute, certes un peu trop curieux, qui enregistrera sur son ordinateur une vidéo de happy slapping via des plates-formes telles que YouTube ou Daily Motion, courra probablement le risque d'être condamné pour recel de diffusion d'images de violence. Ce point devra être précisé par le juge.

S'agissant de la diffusion des images d'agression, pouvaient s'appliquer là aussi des qualifications déjà existantes : ainsi, l'infraction de diffusion aux mineurs d'un message violent ou pornographique (art. 227-24 CP). Les images d'agression rentrent dans cette qualification. Les dispositions de la loi du 21 juin 2004 pour la confiance dans l'éco- nomie numérique permettent la responsabilité pénale des hébergeurs de site diffusant ces images si, ayant connaissance de ces contenus illicites, ils n'ont pas agi avec promptitude pour les retirer ou en rendre l'accès impossible ${ }^{11}$.

\section{Les violences concernées}

Deux groupes de familles d'atteinte à l'intégrité de la personne sont visés : les violences volontaires, à l'exclusion des violences légères contraventionnelles ( $R$ 624-1 et 625-1 $\mathrm{CP}$ ) alors que ces dernières visent pourtant des gifles, des coups, ou des bousculades qui pourraient correspondre à des faits moins graves de happy slapping. Le second groupe vise des infractions relatives aux agressions sexuelles (viol notamment) exceptés l'exhibition sexuelle et le harcèlement sexuel.

Ne sont donc concernées que certaines des violences à l'intégrité de la personne. Ne sont pas incriminés l'enregistrement et la diffusion d'images de violences volontaires à la vie. Le texte exclut de son champ d'application a priori les snuffs movies. Ce sont des films qui mettent en scène un meurtre (supposément) réel. En tout état de cause, se pose la question de savoir pourquoi les atteintes à la vie sont laissées hors champ... Filmer un meurtre est certainement plus condamnable que de filmer une simple dispute. Voilà le reflet de la conception factuelle de nouveaux comportements délictueux. Le choix des infractions qu'il est interdit de filmer et de diffuser est inspiré des premiers cas répercutés dans la presse. Ainsi l'accent est mis sur la nouvelle méthode de légiférer en temps réel, ou coup par coup...

Inversement, ont été inclues des infractions de violence qui déstabilisent. Voilà un autre défaut qui affecte le texte. En effet, le champ d'application des dispositions anti-happy slapping couvre des violences parmi lesquelles se trouvent celles exercées «par une personne dépositaire de l'autorité publique ou chargée d'une mission de service public dans l'exercice ou à l'occasion de l'exercice de ses fonctions ou de sa mission ». Dès lors, un individu qui filmerait une bavure policière serait coupable au titre de la complicité de ces mêmes violences... C'est à ce stade que les limites à la création d'un cas de complicité spécial prennent tout leur relief. En effet, privé des garde-fous que constituent les critères rationnels et éprouvés de la complicité traditionnelle énoncés par l'article 121-7 du Code pénal, l'article 222-33-3 peut mener à une absurdité : celle de devenir complice d'un acte dont on est témoin ou même principal instrument de dénonciation. Surtout, un tel mécanisme est extrêmement nuisible à la liberté de communication qui, si besoin est de le rappeler, constitue une liberté de première génération acquise initialement contre l'État. Être sanctionné en raison de la dénonciation de bavures policières relève de l'ingérence manifeste de l'État dans la liberté du citoyen. Il faudra mesurer ce constat.

10. Parfois même, cette qualification est plus sévère que le système d'emprunt de criminalité mis en place par l'article 222-33-3 CP. C'est ainsi le cas pour l'enregistrement de violences entraînant une ITT de plus de huit jours puisque ces dernières sont punies de trois ans d'emprisonnement et 45000 euros d'amende alors que la non-assistance à personne en péril est punie de cinq ans d'emprisonnement et 75000 euros d'amende. L'objectif du législateur n'est alors pas ici atteint.

11. Voir infra, II, p. 55 
En définitive, il convient de rester mesuré sur l'opportunité de ces nouvelles incriminations et surtout sur la qualité du travail législatif, notamment au regard des violences concernées.

\section{B. L'exception à la répression : un fait justificatif né d'une logique de protection}

Le texte dispose enfin que l'enregistrement et la diffusion ne sont pas sanctionnés dès lors qu'ils sont réalisés dans le cadre de la liberté d'information ou dans un but probatoire (alinéa 3, art. 222-33-3 CP). Il s'agit d'un fait justificatif tiré de la permission de la loi. «Le présent article n'est pas applicable lorsque l'enregistrement ou la diffusion résulte de l'exercice normal d'une profession ayant pour objet d'informer le public ou est réalisé afin de servir de preuve en justice».

\section{L'exception d'information}

En vertu de la liberté d'information, les professionnels de l'information peuvent reproduire l'image de l'individu : le droit à l'image cède devant la nécessité d'informer le public, lorsque se produit un évènement d'actualité tel qu'un fait divers. Cette immunité n'est accordée que sous réserve des dispositions de la loi du 29 juillet 1881 sur la liberté de la presse et plus spécialement sur les articles 35 quater ( «la diffusion par quelque moyen que ce soit et quel qu'en soit le support de la reproduction des circonstances d'un crime ou d'un délit, lorsque cette reproduction porte atteinte gravement à la dignité d'une victime et sans accord de cette dernière est punie de 15000 euros d'amende») et 39 quinquies ( «le fait de diffuser par quelque moyen que ce soit et quel qu'en soit le support des renseignements concernant l'identité d'une victime d'une agression ou d'une atteinte sexuelle ou l'image de cette victime lorsqu'elle est identifiable est puni de 15 ooo euros d'amende »). Autrement dit, l'enregistrement et la diffusion de telles agressions ne doivent porter atteintes ni à la dignité de la victime ni permettre son identification.

\section{L'exception probatoire}

La loi autorise également une personne à filmer une scène d'agression si l'enregistrement est réalisé dans le but d'établir la matérialité des faits ou de faciliter l'identification des auteurs. Cela semble ne s'appliquer qu'à l'enregistrement.

La seconde de ces exceptions, hypothèse où l'enregistrement sert de preuve en justice, pourrait bien servir de palliatif aux dérives évoquées liées aux deux alinéas précédents en matière de condamnation des individus ayant notamment filmé des violences policières. Ceux-ci ne seront pas poursuivis dès lors qu'une plainte est déposée à l'encontre des policiers en faute. Néanmoins, ce remède connaît très vite ses limites en ce sens que la responsabilité des personnes à l'origine de l'enregistrement serait dépendante du dépôt d'une plainte par la victime qui seule permettrait de les exonérer. En outre, l'alinéa 3 précise bien que l'enregistrement doit être réalisé afin de «servir » de preuve en justice. Qu'advient-il alors de l'hypothèse où l'enregistrement sert aussi à des fins de dénonciation dans les médias ou de reportage sur la police? C'est peut-être ici que la première exception de l'alinéa 3 intervient. Le législateur a, en effet, souhaité que l'enregistrement ou la diffusion résultant de l'exercice normal de la profession de journaliste ayant pour objet d'informer le public soient soustraits des sanctions des dispositions contre le happy slapping. Dès lors, la liberté d'expression est sauvegardée et l'économie de l'article 222-33-3 du Code pénal est globalement satisfaisante. Cependant, cette exception en faveur des journalistes introduit une discrimination à l'encontre des individus dépourvus de la fameuse carte de presse. Or, la frontière entre le journaliste «professionnel » et le journaliste occasionnel que l'on rencontre le plus souvent sur Internet est loin d'être évidente. Ce constat maintes fois décrit $^{12}$ rend de plus en plus artificielle la définition du journaliste telle qu'elle figure à l'article L.761-2 du Code du travail ${ }^{13}$. Dès lors, est-il vraiment souhaitable de s'appuyer sur une légitimité à toute épreuve du journaliste dans l'enregistrement de scènes de violences et de présumer l'intention coupable des autres individus? Marquant une vision tronquée de la réalité, cette présomption de culpabilité est une source inépuisable de situations confuses. En effet, qu'en sera-t-il du journaliste qui diffusera la vidéo d'un particulier qui a filmé une bavure policière? Enfin, cette distinction entre le journaliste au sens traditionnel du terme et le particulier, reporter en herbe, vient en contradiction avec une jurisprudence bien assise de la Cour européenne des droits de l'homme. En effet, dans l'arrêt «Çetin et autres c. Turquie » ${ }^{14}$, les juges de Strasbourg ont énoncé que "l'article 10 garantit la liberté d'expression à “toute personne”; il ne distingue pas d'après la nature du but recherché ni d'après le rôle que les personnes, physiques ou morales, ont joué dans l'exercice de cette liberté». Dès lors, cette exception en faveur des seuls journalistes met le législateur en porte-à-faux par rapport aux juges européens qui n'hésiteront pas à le sanctionner dès qu'une occasion se présentera.

L'entrée du happy slapping dans le Code pénal français répond à une exigence de répression, laquelle est immédiatement ébranlée dans le corps même du texte incriminateur eu égard à certaines situations particulières. La logique de protection reprend alors ses droits. Le compromis est alors assuré. Mais, inversement, dans la loi du 5 mars 2007, un certain nombre de mesures préventives prises pour protéger simplement certaines catégories de personnes (les mineurs) convergent indéniablement dans une logique de répression.

12. Notamment L'Opinion numérique, A. Lepage (dir.), Paris, Dalloz (Presaje), 2006, p. 98.

13. Le journaliste professionnel est « celui qui a pour occupation principale, régulière et rétribuée l'exercice de sa profession dans une ou plusieurs publications quotidiennes ou périodiques ou dans une ou plusieurs agences de presse et qui en tire le principal de ses ressources».

14. CEDH, 13 février 2003, "Çetin et autres c. Turquie", $n^{\text {os }} 40153$ / 98 et 4016o / 98, deuxième section, p. 11. 


\section{Les impasses du compromis: le dispositif de protection des mineurs au service d'une meilleure répression des acteurs de la prévention}

La mobilisation des acteurs de la prévention contenue dans la loi du 5 mars 2007 a pour conséquence de leur faire supporter un risque pénal non négligeable. Cette mobilisation se manifeste aussi bien par de nouvelles obligations des acteurs du réseau (A), que par une extension des prérogatives octroyées aux acteurs étrangers au réseau (B).

\section{A. De nouvelles obligations à la charge des intermédiaires techniques ${ }^{15}$}

Dès l'élaboration de la loi du 21 juin 2004, l'Assemblée nationale a voulu renforcer le dispositif d' « autodiscipline professionnelle » pour les délits de pédophilie, d'incitation à la haine raciale et d'apologie des crimes de guerre. Ce choix s'est traduit par un problématique article 6-I-7 dans la loi pour la confiance dans l'économie numérique qui, tout en rappelant l'absence d'obligation générale de surveillance à la charge des intermédiaires techniques, impose leur concours à la lutte contre les plus graves dérives sur Internet : pornographie infantile, incitation à la haine raciale, apologie de crimes contre l'humanité. Hébergeurs et intermédiaires techniques doivent prévoir un dispositif de signalement et informer les autorités de tout contenu de ce type qui leur serait ainsi signalé. Ce bel équilibre n'aura pas duré trois ans. Depuis, il semble que le législateur se soit aperçu que la liste des infractions les plus graves était trop succincte au regard des évolutions des pratiques sur Internet. Dès lors, les obligations ciblées de surveillance à la charge des intermédiaires techniques se sont élargies au regard tant de la violence qu'à celui des jeux d'argent illicites.

\section{La lutte contre l'incitation à la violence et les atteintes à la dignité}

Dans sa rédaction antérieure à la loi du 5 mars 2007, l'article 6-I-7 alinéa 3 disposait que, «compte tenu de l'intérêt général attaché à la répression de l'apologie des crimes contre l'humanité, de l'incitation à la haine raciale ainsi que de la pornographie enfantine, les personnes mentionnées ci-dessus doivent concourir à la lutte contre la diffusion des infractions visées aux cinquième et huitième alinéas de l'article 24 de la loi du 29 juillet 1881 sur la liberté de la presse et à l'article 227-23 du Code pénal ». L'article 40 de la loi sur la prévention de la délinquance ajoute la répression de «l'incitation à la violence » et des « atteintes à la dignité humaine » dans la sphère de l'intérêt général et impose aux intermédiaires techniques de concourir à la lutte contre une nouvelle infraction, à savoir celle de l'article 227-24 du Code pénal. Ce dernier sanctionne le « fait soit de fabriquer, de transporter, de diffuser par quelque moyen que ce soit et quel qu'en soit le support un message à caractère violent ou pornographique ou de nature à porter gravement atteinte à la dignité humaine, soit de faire commerce d'un tel message [...] lorsque ce message est susceptible d'être vu ou perçu par un mineur ».

De prime abord, il est judicieux de penser que ces nouvelles obligations imposées par l'article 6-I-7 de la LCEN seraient en relation directe avec le nouvel article 222-33-3 du Code pénal sanctionnant le happy slapping. Ainsi, le législateur aurait souhaité accompagner cette nouvelle incrimination par une obligation spéciale de surveillance des intermédiaires techniques en la matière. Un tel choix aurait été signe de l'efficacité avec laquelle les parlementaires ont voulu endiguer ce phénomène nouveau. Toutefois, une telle interprétation néglige le fait que, dans l'article 6-I-7 nouveau, la répression de «l'incitation à la violence » et des «atteintes à la dignité humaine » est directement rattachée à l'article 227-24 du Code pénal. Ainsi, le législateur délimite les obligations des intermédiaires techniques aux messages qualifiés d'illicites dès lors qu'ils sont susceptibles d'être vus pas des mineurs. Or, les images de violence enregistrées via le happy slapping ne relèvent pas de cette catégorie et sont illicites quel que soit le public qui les visionne. Pourtant, il aurait peutêtre été nécessaire de les ajouter dans cet article 6-I-7 censé protéger l'intérêt général.

Quoi qu'il en soit, c'est bien le mineur que les parlementaires ont souhaité protéger. D'ailleurs, cette modification de l'article 6-I-7 fut introduite par un amendement du gouvernement dont l'exposé est explicite : «Le Gouvernement est pleinement conscient des dérives parfois inqualifiables de certains jeux vidéos, dont l'ultra violence va souvent de pair avec une absence totale d'interdit. Ce type de jeu peut contribuer à supprimer toute limite et tout repère par rapport à la vie réelle, notamment par la représentation ludique de certains actes odieux tels que viols ou meurtres ». Dès lors, cette obligation des intermédiaires techniques s'accorde avec l'article 35-I de la loi sur la prévention de la délinquance qui vise à renforcer la législation relative au contrôle des documents pouvant présenter un danger pour la jeunesse en raison de leur caractère pornographique ou de la place qui y est faite à la violence. L'article 35-I de la loi modifie ainsi les articles 32 à 39 de la loi no $98-468$ du 17 juin 1998 relative à la prévention et à la répression des infractions sexuelles ainsi qu'à la protection des mineurs. Il est précisé que dans le cas d'un document dangereux en raison de son caractère pornographique, le support et chaque unité de son conditionnement doivent comporter de façon lisible, visible et inaltérable, la mention «mise à disposition des mineurs

15. C'est-à-dire les intermédiaires qui transmettent ou hébergent des informations émanant de tiers - ils se caractérisent par l'accomplissement d'une tâche technique entre l'envoi de données et la réception finale des informations. Le trait commun de tous ces intermédiaires est de ne pas exercer de droit de regard sur l'information qui transite grâce à eux - fonction d'hébergement, c'est-à-dire le stockage et le traitement de données à la demande de l'utilisateur du service sur le système du prestataire. 
interdite ». La mise en œuvre de cette obligation incombe à l'éditeur ou à défaut au distributeur chargé de la diffusion en France du document. Cette mention emporte interdiction de proposer, donner ou louer ou vendre le produit en cause aux mineurs, la transgression intentionnelle de ce texte étant punie d'un an d'emprisonnement et de 15 ooo euros d'amende. L'objectif est de renforcer l'application du principe général d'interdiction de mise à disposition de contenus pornographiques aux mineurs fixé à l'article 227-14 CP. Aussi, l'alinéa 2 de l'article 32 de la loi de 1998 dispose que «lorsqu'un document fixé par un procédé identique peut présenter un risque pour la jeunesse en raison de la place faite au crime, à la violence, à l'incitation à l'usage, à la détention ou au trafic de stupéfiants, à l'incitation à la consommation excessive d'alcool ainsi qu'à la discrimination ou à la haine contre une personne déterminée ou un groupe de personnes, le support et chaque unité de son conditionnement doivent faire l'objet d'une signalétique spécifique au regard de ce risque. Cette signalétique, dont les caractéristiques sont fixées par l'autorité administrative (v. supra), est destinée à en limiter la mise à disposition à certaines catégories de mineurs, en fonction de leur âge ». Le législateur a profité de la seconde lecture pour corriger la maladresse de rédaction de l'alinéa 2 de l'article 32 de la loi de 1998 et permet ainsi de viser les « documents fixés par un procédé déchiffrable par voie électronique en mode analogique ou en mode numérique (l'ancienne version du texte visait « tout support et unité de conditionnement mentionnés au premier alinéa », c'est-à-dire uniquement ceux présentant un contenu pornographique). Par exemple, un document présentant un risque pour la jeunesse en raison de la place faite à l'incitation à la consommation excessive d'alcool entre dorénavant dans le champ d'application de cet article. Dès lors, après signalement, les intermédiaires techniques devront informer promptement les autorités compétentes de tout site pornographique ou incitant à la violence, dénué de mesures de protection destinées à empêcher les mineurs d'y avoir accès. On imagine les perspectives qu'ouvrent les possibles interprétations de notions dont l'objectivité laisse songeur; les œuvres de Donatien Alphonse de Sade, non seulement en vente libre mais par surcroît du domaine public, devraient-elles être précédées d'un avertissement, voire d'accès restreint? Pourra-t-on licitement diffuser une reproduction de l'Origine $d u$ monde de Courbet? Tout donne à penser que ces chefs-d'œuvre d'hier continueront d'être librement mis en ligne; mais leurs équivalents d'aujourd'hui?

\section{La lutte contre les activités illicites de jeu d'argent}

Le nouveau texte n'a en tout cas plus rien à voir avec le cadre précis et restreint de l'exception au principe dans son état actuel, lequel résultait d'un accord sur des objectifs et acceptions partagés par l'ensemble des pays membres de l'Union, transposé tel quel par la LCEN. Mieux encore, la nouvelle loi trouve dans « l'intérêt général attaché à la répression des activités illégales de jeux d'argent » matière à une nouvelle dérogation au principe de nonsurveillance de l'Internet par ses intermédiaires techniques. Or, il n'est pas évident que cette nouvelle obligation à la charge des intermédiaires techniques ait une longue espérance de vie. Ainsi que l'a souligné Monsieur le Député Claude Goasguen dans un amendement rejeté: «la circulaire du Premier ministre du 9 décembre 1999, qui précise les conditions de transposition des directives communautaires $n^{\circ} 98$ / 34 / CE et 98 / 48 / CE, prévoit que toutes les règles nationales visant spécifiquement les services de la société de l'information devront être soumises aux instances communautaires avant d'être adoptées ». Dès lors, ce nouvel alinéa ajouté à l'article 6-I-7 LCEN aurait dû être notifié à la Commission européenne avant d'entrer en vigueur. Néanmoins, la Commission des lois a rejeté cet amendement et considère que seul le décret d'application de cette disposition devra être soumis aux instances communautaires. Cette position ne change pourtant pas outre mesure l'effectivité de l'obligation des intermédiaires. En premier lieu, elle suppose que le décret d'application ne reste pas lettre morte, ce qui est loin d'être garanti. En second lieu, la notification du décret à la Commission européenne fait courir un délai dit de «statu quo » pendant lequel les règles projetées ne peuvent pas être mises en vigueur. Ce délai peut être très long. Autant dire une éternité en droit de l'Internet surtout si l'on y ajoute le délai susceptible de s'écouler avant la parution du décret... Par ailleurs, si cette obligation paraît envisageable pour les fournisseurs d'accès, elle semble très inadaptée aux hébergeurs. En quoi ces derniers doivent-ils informer des internautes avec lesquelles ils n'ont aucun lien contractuel? Concernant les fournisseurs d'accès, cette obligation peut se concevoir en ce sens qu'elle s'inscrirait implicitement dans une relation contractuelle leur imposant d'informer leurs abonnés au sujet des sites de jeu illégaux et des peines encourues du fait d'actes de jeu. Même en se plaçant dans la relation contractuelle entre l'hébergeur et ses clients - à savoir des éditeurs de sites - il paraît difficile d'envisager en quoi l'hébergeur devrait leur fournir une liste de sites de jeux illicites alors que son service se limite à un stockage, et non à une connexion au réseau. En outre, signe sans doute révélateur de l'intention du législateur, les débats parlementaires ne mentionnent presque systématiquement que les seuls fournisseurs d'accès et non les hébergeurs. Le rôle des intermédiaires techniques dans la lutte contre les jeux d'argent illicites n'est donc pas prêt de se concrétiser. D'ailleurs, ce constat est peut-être une bonne chose. Il semble en effet que l'application de cet article 40 pourrait avoir l'effet inverse en ce sens que la fourniture de la liste des sites de jeux illégaux constituerait une sorte de publicité en leur faveur...

L'article 36 de la loi du 5 mars dernier modifie l'article 3 de la fameuse loi du 21 mai $18366^{16}$ par un doublement de la peine encourue par l'organisateur de loteries prohibées passant de 30000 à 60000 euros. En outre, parmi les dispositions relatives aux jeux, il convient aussi de mentionner l'article $38-\mathrm{V}-1$ de cette loi sur la prévention de la 
délinquance qui ajoute à l'article $1^{\mathrm{er}}$ de la loi du 12 juillet $1983^{17}$ la disposition suivante : « le fait de faire de la publicité, par quelque moyen que ce soit, en faveur d'une maison de jeux de hasard non autorisée est puni de 30000 euros d'amende. Le tribunal peut porter le montant de l'amende au quadruple du montant des dépenses publicitaires consacrées à l'opération illégale ». Ainsi, les annonceurs se voient une nouvelle fois visés dans la lutte contre la délinquance sur les réseaux.

Ce choix législatif d'un alourdissement des sanctions intervient dans un climat où les instances européennes sont de plus en plus méfiantes face à la défense des États membres de leurs monopoles en matière de jeu. Le lendemain de la promulgation de la loi sur la prévention de la délinquance, la Cour de justice des Communautés européennes a rendu une décision à propos des sanctions pénales italiennes en cas d'organisation de jeux de hasard ou de collecte de paris sans avoir obtenu une autorisation ou une concession préalable ${ }^{18}$. Dans cette affaire Placanica, les juges européens sanctionnent explicitement l'Italie qui, sous couvert de l'intérêt public, protège son monopole sur les jeux au détriment des acteurs étrangers. Dès lors, il y a de grands risques pour que les juges de La Haye observent une position similaire face au dispositif pénal français.

Toutes ces mesures montrent à quel point les principes européens liés à la non-surveillance du réseau par les intermédiaires techniques irritent le législateur incapable d'accepter la transnationalité structurelle de l'Internet.

\section{L'autorité chargée de prononcer les interdictions et de fixer les caractéristiques de la signalétique}

L'ancien article 32 de la loi de 1998 prévoyait que les mesures d'interdiction étaient prononcées par une autorité administrative après avis consultatif d'une commission administrative. L'article 33, tel que modifié par la loi, mentionne la seule autorité administrative et supprime donc l'intervention de la commission administrative. Cette modification de la procédure aligne celle-ci sur le dispositif de la loi du 16 juillet 1949, qui confère au ministre de l'Intérieur le pouvoir d'interdire certaines publications aux mineurs «en raison de leur caractère licencieux ou pornographique, ou de la place faite au crime, à la violence, à la discrimination ou à la haine raciale, à l'incitation à l'usage, à la détention ou au trafic des stupéfiants ». La loi prévoit en outre que l'autorité administrative fixe les caractéristiques de la signalétique de classification des documents présentant un risque pour la jeunesse. Compte tenu du caractère sensible et évolutif des questions en cause, il serait souhaitable que ces caractéristiques soient définies en fonction des supports et des types de documents, après une concertation de l'ensemble des acteurs concernés. Le texte prévoit que cette signalétique prendra en considération «l'âge» des mineurs, ce qui ne manquera pas de réveiller les éternels débats sur le bien-fondé des catégories d'âge. La loi laisse également la possibilité à l'autorité administrative d'interdire la mise à disposition des mineurs de documents à caractère préjudiciable, même s'ils respectent les obligations fixées en matière de signalétique. Le législateur a donc jugé nécessaire de prévoir que l'interdiction de documents dangereux pour la jeunesse pourra intervenir y compris lorsqu'une signalétique existe.

Si l'on comprend l'objectif de cette signalétique qui permettra aux consommateurs et aux vendeurs de repérer les produits présentant un risque pour la jeunesse et d'ajuster leur propre comportement, il reste que le ministre devra prendre en compte les éventuelles difficultés d'articulation de celle-ci avec l'existant. Le risque de multiplication des signalétiques est réel et peut s'avérer contreproductif en créant une confusion dans l'esprit du consommateur. On pense notamment, en matière de jeux vidéo, au système PEGI (Pan European Game Information).

Cette question ne se posera pas en revanche s'agissant des œuvres cinématographiques puisque l'article 35 précise que « les dispositions du présent chapitre ne s'appliquent pas aux documents qui constituent la reproduction intégrale d'une œuvre cinématographique ayant obtenu le visa prévu à l'article 19 du code de l'industrie cinématographique». Seule exception en la matière, les films pornographiques ou incitant à la violence au sens de la loi du 30 décembre 1975 (articles 11 et 12) qui eux seront bien soumis de plein droit à l'interdiction prévue au premier alinéa de l'article 32.

En définitive, les notions extrêmement larges de dignité humaine et d'incitation à la violence doivent être interprétées à la lumière de l'article 227-24 du Code pénal. Toutefois, cette nouvelle obligation à la charge des intermédiaires techniques n'en est pas pour autant minime. En effet, à titre d'illustration, l'International Watch Foundation (IWF) a comptabilisé aux alentours de 27000 signalisations de tels sites illicites sur l'année 2006. Dès lors, il ne faudrait pas multiplier encore les cas pour lesquels les intermédiaires techniques doivent concourir à l'intérêt général, même si la « santé » du réseau est sans doute à ce prix.

\section{B. De nouvelles prérogatives pour des acteurs étrangers aux réseaux}

Le renforcement des obligations à la charge des intermédiaires techniques était sans aucun doute nécessaire mais absolument pas suffisant. En effet, la nouvelle politique de répression ne peut s'appuyer seulement sur des acteurs appartenant au réseau. Ainsi, les officiers ou agents de polices judiciaires connaissent des moyens supplémentaires pour constater et recueillir la preuve d'infractions sur Internet tandis que les établissements financiers peuvent être requis de geler les fonds des organisateurs de jeux illicites. 


\section{Constats et preuves des infractions par les cyber-patrouilleurs}

Dans le souci constant de protection des mineurs dont le législateurs a fait preuve lors de l'élaboration de la loi du 5 mars 2007, un renforcement des prérogatives des officiers de police judiciaire - ou « cyber-patrouilleurs » tels qu'ils ont été surnommés par un parlementaire - a été décidé. Ainsi, dans deux titres différents du Code de procédure pénale, l'article 35-III de la loi sur la prévention de la délinquance insère deux articles identiques (art. 70647-2 et 706-47-3 cpp) aux termes desquels : «Dans le but de constater les infractions mentionnées aux articles 2254-1 à 225-4-9, 225-5 à 225-12 et 225-12-1 à 225-12-4 du Code pénal (Du recours à la prostitution de mineurs ou de personnes particulièrement vulnérables) [et aux articles 22718 à 227-24 - De la mise en péril des mineurs] et, lorsque celles-ci sont commises par un moyen de communication électronique, d'en rassembler les preuves et d'en rechercher les auteurs, les officiers ou agents de police judiciaire agissant au cours de l'enquête ou sur commission rogatoire peuvent, s'ils sont affectés dans un service spécialisé et spécialement habilités à cette fin, dans des conditions précisées par arrêté, procéder aux actes suivants sans en être pénalement responsables: $1^{\circ}$ Participer sous un pseudonyme aux échanges électroniques; $2^{\circ}$ Etre en contact par ce moyen avec les personnes susceptibles d'être les auteurs de ces infractions $; 3^{\circ}$ Extraire, transmettre en réponse à une demande expresse, acquérir ou conserver des contenus illicites dans des conditions fixées par décret. À peine de nullité, ces actes ne peuvent constituer une incitation à commettre ces infractions ».

Le dernier alinéa de cet article 35-III visant à prévenir toute incitation par les policiers à commettre les infractions visées est sans aucun doute significatif de toutes les difficultés soulevées par ces nouvelles prérogatives. Comme l'a très justement remarqué Monsieur le Député Houillon, « le rôle des enquêteurs doit se limiter à un rôle d'observateurs des échanges de contenus illicites, pour éviter qu'ils ne puissent être accusés d'avoir sollicité de quelque ma-nière que ce soit un comportement illicite ${ }^{19}$. Ainsi, la précision suivante: "en réponse à une demande expresse ", aurait été ajoutée pour éviter toute équivoque et cantonner les cyber-patrouilleurs à un rôle purement passif.

L'enjeu est de taille puisque la Chambre criminelle de la Cour de cassation a posé le principe suivant en la matière: « porte atteinte au principe de la loyauté des preuves et au droit à un procès équitable, la provocation à la commission d'une infraction par un agent de l'autorité publique ou par son intermédiaire; que la déloyauté d'un tel procédé rend irrecevables en justice les éléments de preuve ainsi obtenus ${ }^{20}$. En l'espèce, un individu avait, à l'instigation de policiers, incité le prévenu à lui transmettre des images de mineurs présentant un caractère pornographique. La Cour de cassation avait alors considéré que la déloyauté du procédé originaire annulait les aveux ultérieurs du prévenu au sujet de sa détention d'images illicites sur son disque dur. Pourtant, cette détention d'images n'avait pas été provoquée par une quelconque incitation de la part des policiers. Dès lors, la provocation illicite a pour conséquence une nullité de tous les actes de procédures consécutifs, même ceux relatifs à des infractions commises antérieurement à l'intervention des officiers de police judiciaire. Cette contamination de l'illicéité de la provocation à l'ensemble de la procédure ne laisse aux policiers aucun droit à l'erreur au cours de leurs enquêtes. Toutefois, si la jurisprudence exècre toute provocation à l'infraction, elle tolère la provocation à la preuve d'agissements délictueux déjà commis ou en train de se commettre $^{21}$. Les officiers de police judiciaire devront alors mener leurs enquêtes à la lumière de cette rigueur exigeante que leur imposent les juges. Ainsi, leurs nouvelles prérogatives dans la traque aux cyber-délinquants se limiteront à être en contact et échanger des informations avec ces derniers dans le strict objectif de collecter des preuves sur des infractions commises ou en cours et sans jamais influer sur les choix des délinquants.

Pourtant, à la lecture d'autres dispositions de la loi sur la prévention de la délinquance, il semble que le législateur attende plus des policiers. En effet, ce nouveau dispositif en faveur des officiers de police judiciaire vient à la suite de l'article $35-\mathrm{II}^{22}$ insérant une nouvelle infraction sanctionnant le fait pour un majeur, via Internet, de faire des propositions sexuelles à un mineur de 15 ans ou à « une personne se présentant comme telle ${ }^{23}$. Cette dernière

19. P. Houillon, Rapport $n^{\circ} 3674$, p. 53.

20. Cass. crim., 11 mai 2006, $\mathrm{n}^{\circ}$ 05-84.837 : Juris-Data n ${ }^{\circ}$ 2006-033840; CCE, janvier 2007, comm. $\mathrm{n}^{\circ}$ 12, obs. Lepage; AJ Pénal, septembre 2006, p. 354, obs. Vergès.

21. Cass. crim., $1^{\text {er }}$ octobre 2003, $\mathrm{n}^{\circ}$ 03-84.142 : Juris-Data $\mathrm{n}^{\circ}$ 2003-020653; D, 2004, jurisp. P.1845, obs. Sanchez.

22. Cet article vient compléter une insuffisance regrettable du droit français pour prévenir la pédophilie sur le réseau. En effet, premièrement, l'article $227-26$ $4^{\circ}$ du Code pénal porte certes à dix ans d'emprisonnement et 150 ooo euros d'amende l'infraction prévue à l'article précédent à savoir «le fait, par un majeur, d'exercer sans violence, contrainte, menace ni surprise une atteinte sexuelle sur la personne d'un mineur de quinze ans» lorsque celle-ci est commise via un réseau de télécommunication. Néanmoins, l’infraction est bien évidemment qualifiée seulement à partir du moment où l'acte est accompli. Le nouvel article 227-22-1 s'inscrit donc dans une logique de prévention de la pédophilie sur les réseaux en sanctionnant dès le stade des propositions sexuelles du majeur sur le mineur de 15 ans. Deuxièmement, cette nouvelle incrimination est aussi une réponse à l'analyse jurisprudentielle restrictive de la provocation de mineur à la débauche prévue et réprimée par l'article 227-22 du Code pénal. Dans un arrêt du 14 novembre 1990, la Chambre criminelle a ainsi affirmé que l'excitation de mineur à la débauche n'est pénalement répréhensible que si l'auteur des faits a eu en vue la perversion de la jeunesse, et pas seulement la satisfaction de ses propres passions. D'ailleurs, cette décision s'accorde avec une très ancienne rendue par les Chambres réunies de la Cour de cassation le $1^{\mathrm{er}}$ mai 1854 qui avaient exclu l'application du texte lorsque l'agent a simplement cherché à séduire personnellement et directement sa victime (Cass. ch. réun., $1^{\mathrm{er}}$ mai 1854, D.P., 1854.I.261, $2^{\mathrm{e}}$ espèce). Ainsi, une nouvelle fois, les sanctions pénales étaient trop limitées dans leur application pour lutter efficacement contre la pédophilie en ligne.

23. Cette précision démontre que le législateur a souhaité punir, en premier lieu, la mens rea - ou volonté criminelle - puisqu’il suffit que l'agent ait cru faire des propositions sexuelles à un mineur de quinze ans pour se voir condamner pénalement. En outre, il semble que cette précision relève d'une 
hypothèse prévue par le législateur n'a-t-elle pas pour fin de permettre aux policiers de constater sur le champ une infraction qu'ils ont provoquée en se faisant passer pour un mineur? En effet, l'on ne voit pas bien en quoi le législateur souhaiterait sanctionner un individu faisant des avances à un adulte. On est bien dans la provocation à l'infraction. Pour autant, cette hypothèse correspond en fait à une tentative punissable au même titre que l'infraction consommée. Ainsi, à l'instar de l'agent tentant de tuer une personne déjà morte, le majeur faisant des avances à un " faux mineur» demeure au stade de la tentative en raison de l'impossibilité de l'infraction. En droit pénal général, cette impossibilité de l'infraction est un élément indépendant de la volonté de l'agent et demeure alors punissable en tant que telle. Mais pourquoi avoir envisagé ce cas alors même que le régime général de la responsabilité pénale permettait une sanction ? À n'en pas douter, le législateur pousse, si ce n'est de façon directe du moins inconsciemment, les cyber-patrouilleurs à la faute et par là même trahit ses intentions. Dès lors, les nouvelles prérogatives attribuées aux officiers de police judiciaire appellent une prudence sans réserve.

\section{Gel des fonds des organisateurs de jeux illicites par les établissements financiers}

Un des meilleurs outils pour lutter contre la délinquance, et notamment les jeux et paris en ligne illicites, consiste à s'attaquer à la base, à savoir les gains. Pour ce faire, le législateur a sollicité l'aide des établissements financiers. Ainsi l'article 36 de la loi du 5 mars 2007 crée notamment deux articles dans le Code monétaire et financier. En premier lieu, l'article L.565-1 du CMF dispose que « les organismes, institutions et services régis par le titre $\mathrm{I}^{\mathrm{er}} \mathrm{du}$ présent livre qui détiennent ou reçoivent des fonds du public sont tenus d'appliquer les mesures d'interdiction prises en vertu du présent chapitre ». En second lieu, l'article L.565-2 du même Code précise que «le ministre chargé des finances et le ministre de l'intérieur peuvent décider d'interdire, pour une durée de six mois renouvelable, tout mouvement ou transfert de fonds en provenance des personnes physiques ou morales qui organisent des activités de jeux, paris ou loteries prohibés par la loi du 21 mai 1836 portant prohibition des loteries et la loi du 2 juin 1891 ayant pour objet de réglementer l'autorisation et le fonctionnement des courses de chevaux, ainsi que la loi n ${ }^{\circ} 83^{-}$ 628 du 12 juillet 1983 relative aux jeux de hasard».

Ce mécanisme de gel des fonds par les établissements bancaires n'est pas sans rappeler celui introduit par la loi du 23 janvier 2006 relative à la lutte contre le terrorisme ${ }^{24}$.
Dans un avis relatif à cette dernière loi, le Conseil d'État avait considéré qu'une mesure de gel ou d'interdiction qui prive temporairement, à des fins d'ordre public précisément identifiées, un titulaire de compte, du droit de disposer des fonds qui lui appartiennent, n'équivaut pas à une dépossession et n'est pas susceptible de porter atteinte aux libertés individuelles ${ }^{25}$. Ainsi, les mesures, prises en application du nouvel article L.565-2 CMF qui prévoit une durée de 6 mois renouvelable, respectent les libertés individuelles des organisateurs de jeux par son caractère temporaire. Il convient de noter que l'article 36 de la loi sur la prévention de la délinquance insère aussi un article L.5654 en vertu duquel: «l'État est responsable des conséquences dommageables de la mise en œuvre de bonne foi, par les organismes, institutions et services régis par le titre $\mathrm{I}^{\mathrm{er}}$ du présent livre, leurs dirigeants ou leurs préposés, des mesures d'interdiction mentionnées à l'article L. 565-2. Aucune sanction professionnelle ne peut être prononcée à l'encontre de ces organismes, institutions ou services, leurs dirigeants ou leurs préposés ». Cette limitation de responsabilité à destination des établissements bancaires est la moindre des choses pour ces acteurs impliqués dans la lutte contre les jeux d'argents illicites.

\section{Conclusion}

La prévention, instrument de répression ou de protection? Dans la loi du 5 mars 2007, il est difficile d'y voir clair. Les logiques s'affrontent et font apparaître autant de paradoxes que d'incohérences et d'imprécisions. Une métaphore avec le mythe de la boîte de Pandore permet de donner l'impression générale... Zeus remet à Pandore une boîte, contenant tous les maux de l'humanité enfermés par Prométhée pour en protéger les hommes, notamment la Vieillesse, le Travail, la Maladie, la Folie, le Vice, la Tromperie et la Passion, ainsi que l'Espérance en lui conseillant bien de ne jamais l'ouvrir. Cédant à la curiosité, Pandore ouvre la boîte: elle libère ainsi les maladies et malheurs qu'elle contient. Elle referme la boîte trop tard pour les retenir, et seule l'Espérance, plus lente à réagir, y reste enfermée... La loi du 5 mars 2007 est la boîte de Pandore des juristes qui une fois examinée libère tous les maux redoutés d'une loi: l'imprécision, l'incohérence, la casuistique, le renversement des valeurs, les paradoxes, la contradiction, la complexité... Il ne reste alors qu'un espoir dans l'avenir : celui de ne pas rencontrer une nouvelle boîte de Pandore.

24. Loi n ${ }^{\circ}$ 2006-64 du 23 janvier 2006 relative à la lutte contre le terrorisme et portant dispositions diverses relatives à la sécurité et aux contrôles frontaliers, JO du 24 janvier 2006, p. 1129.

25. C. Cutajar, «Le gel des avoirs terroristes, nouvel outil de lutte contre le financement du terrorisme», JCP G, $\mathrm{n}^{\circ} 20,17$ mai 2006 , act. $\mathrm{n}^{\circ} 214$, p. 984 . 